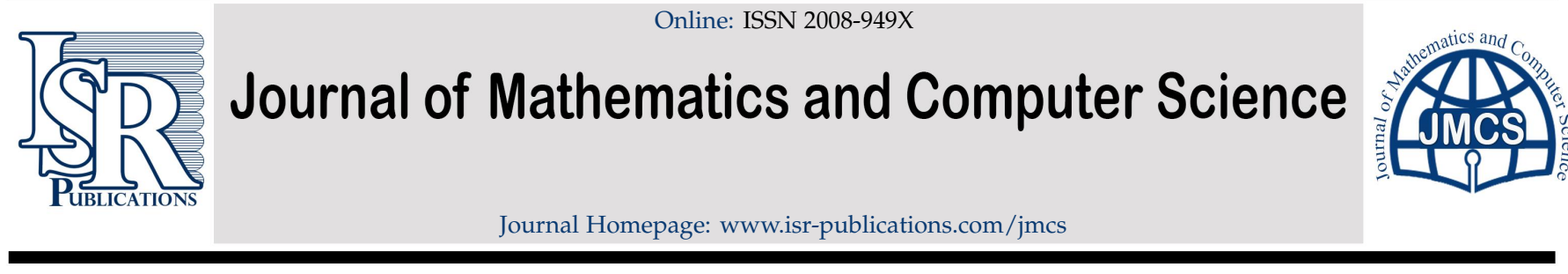

\title{
Computing the edge metric dimension of convex polytopes related graphs
}

\author{
Muhammad Ahsan ${ }^{a}, *$, Zohaib Zahid ${ }^{a}$, Sohail Zafar ${ }^{a}$, Arif Rafiq ${ }^{b}$, Muhammad Sarwar Sindhu $^{\mathrm{b}}$, Muhammad \\ Umarb $^{\mathrm{b}}$
}

${ }^{a}$ Department of Mathematics, University of Management and Technology, Lahore 54770, Pakistan.

${ }^{b}$ Department of Mathematics, Virtual University of Pakistan (VU), Lahore, Pakistan.

\begin{abstract}
Let $G=(V(G), E(G))$ be a connected graph and $d(f, y)$ denotes the distance between edge $f$ and vertex $y$, which is defined as $d(f, y)=\min \{d(p, y), d(q, y)\}$, where $f=p q$. A subset $W_{E} \subseteq V(G)$ is called an edge metric generator for graph $G$ if for every two distinct edges $f_{1}, f_{2} \in E(G)$, there exists a vertex $y \in W_{E}$ such that $d\left(f_{1}, y\right) \neq d\left(f_{2}, y\right)$. An edge metric generator with minimum number of vertices is called an edge metric basis for graph $G$ and the cardinality of an edge metric basis is called the edge metric dimension represented by $\operatorname{edim}(G)$. In this paper, we study the edge metric dimension of flower graph $f_{n \times 3}$ and also calculate the edge metric dimension of the prism related graphs $D_{n}^{\prime}$ and $D_{n}^{t}$. It has been concluded that the edge metric dimension of $D_{n}^{\prime}$ is bounded, while of $f_{n \times 3}$ and $D_{n}^{t}$ is unbounded.
\end{abstract}

Keywords: Edge metric dimension, edge metric generator, edge metric basis, resolving set, prism related graphs, flower graph. 2020 MSC: 05C12, 05C25.

(C)2021 All rights reserved.

\section{Introduction and preliminaries}

Slater proposed the idea of metric dimension to find the location of intruder in a network (see $[18,23])$. The proposed idea was further extended by Melter and Harary in [10]. Metric dimension is important in robot navigation, chemistry, problems of image processing and pattern recognition etc. (see $[4,5,11,12$, $14,15])$. The use of metric dimension of graphs was also observed in games like mastermind and coin weighing (see [3]). Caceres et al. computed the metric dimension of cartesian product of graphs and Hallaway et al. had worked on the metric dimension of permutation of graphs (see [3, 9]). Further Zhang had worked on the theory and resolvability of graphs in [6].

Suppose $G$ is connected graph having edge set $E(G)$ and vertex set $V(G)$, also $|E(G)|$ shows the size of graph $G$ and $|V(G)|$ represents the order of graph $G$. Let $N(a)=\{b \in V(G) \mid a b \in E(G)\}$ denote the neighborhood of the vertex $a$, then $|N(a)|$ is called the degree of the vertex a. Moreover, $\Delta(G)$ and $\delta(G)$ represent the maximum and minimum degree of graph $\mathrm{G}$, respectively.

\footnotetext{
*Corresponding author

Email address: muhammadahsanm@gmail.com (Muhammad Ahsan)

doi: $10.22436 /$ jmcs.022.02.08
}

Received: 2020-05-17 Revised: 2020-06-03 Accepted: 2020-06-16 
The distance between two distinct vertices $s$ and $t$, is the number of edges in a shortest path between them and it is denoted by $d(s, t)$. A vertex $u \in V(G)$ is said to distinguish a pair of vertices $s, t \in V(G)$ if $d(s, u) \neq d(t, u)$. A set $W \subseteq V(G)$ is a metric generator for $G$ if every pair of vertices of $G$ can be distinguished by some vertex in $W$. A metric basis is the minimum metric generator for graph $G$ and number of elements in metric basis is called the metric dimension of $G$, denoted by $\operatorname{dim}(G)$. It was shown that computing the metric dimension of a graph is NP-hard [14].

The edge metric dimension is introduced recently. The concept was brought by kelenc et al. and further studied by Zubrilina, peterin, Kratica, Yuezhong Zhang and Ahsan et al. [1, 7, 13, 16, 22, 23]. We can find the distance between an edge $f=p q$ and a vertex $y$ as follows:

$$
d(f, y)=\min \{d(p, y), d(q, y)\}
$$

A vertex $a \in V(G)$ is said to distinguish two distinct edges $e_{1}, e_{2} \in E(G)$ if $d\left(e_{1}, a\right) \neq d\left(e_{2}, a\right)$. A set $W_{E}$ is an edge metric generator of a graph $G$ if every two distinct edges are distinguished by some vertex of $W_{\mathrm{E}}$. An edge metric basis is the minimum edge metric generator of graph $\mathrm{G}$ and its cardinality is called edge metric dimension, denoted by edim $(G)$.

For an ordered subset $W_{E}=\left\{a_{1}, a_{2}, \ldots, a_{k}\right\}$ of the vertex set of $V(G)$, the k-tuple $r\left(e \mid W_{E}\right)=\left(d\left(e, a_{1}\right)\right.$, $\left.d\left(e, a_{2}\right), \ldots, d\left(e, a_{k}\right)\right)$ is called the edge metric representation of an edge $e$ with respect to $W_{E}$. In this sense, $W_{E}$ is an edge metric generator for $G$ if and only if for every pair of different edges $e_{1}, e_{2}$ of $E(G)$, we have $r\left(e_{1} \mid W_{E}\right) \neq r\left(e_{2} \mid W_{E}\right)$.

In this whole paper, all vertex indices are considered to be module $n$. The propositions given below are very helpful for calculating the edge metric dimension of graphs.

Proposition 1.1 ([13]). If $\mathrm{G}$ is a connected graph, then $\operatorname{edim}(\mathrm{G}) \geqslant\left\lceil\log _{2} \Delta(\mathrm{G})\right\rceil$.

Proposition 1.2 ([13]). If $\mathrm{G}$ is a connected graph, then $\operatorname{edim}(\mathrm{G}) \geqslant 1+\left\lceil\log _{2} \delta(\mathrm{G})\right\rceil$.

In following proposition, we will demonstrate the metric dimension of flower graph.

Proposition 1.3 ([11]). For the flower graph $\mathrm{f}_{\mathrm{n} \times 3}$ with $\mathrm{n} \geqslant 6$, we have

$$
\operatorname{dim}\left(f_{n \times 3}\right)= \begin{cases}2, & n \text { is even } \\ 3, & \text { otherwise }\end{cases}
$$

In the following propositions we calculate the metric dimensions of $D_{n}^{\prime}$ and $D_{n}^{t}$ by showing its resolving sets and the results are obvious.

Proposition 1.4. For the prism related graph $\mathrm{D}_{n}^{\prime}$ with $\mathrm{n} \geqslant 4$, we have $\operatorname{dim}\left(\mathrm{D}_{n}^{\prime}\right)=3$ and its metric basis is $W=\left\{a_{1}, a_{2}, a_{k+2}\right\}$, where either $n=2 k$ or $n=2 k+1$.

Proposition 1.5. For the prism related graph $\mathrm{D}_{\mathrm{n}}^{\mathrm{t}}$ with $\mathrm{n} \geqslant 4$, we have $\operatorname{dim}\left(\mathrm{D}_{\mathfrak{n}}^{\mathrm{t}}\right)=3$ and its metric basis is $W=\left\{a_{1}, a_{2}, a_{k+1}\right\}$, where either $n=2 k$ or $n=2 k+1$.

The rest of paper is structured as follows. In Section 2, edge metric dimension of flower graph $f_{n \times 3}$ will be studied. In Section 3, edge metric dimension of prism related graph $D_{n}^{\prime}$ will be investigated. In Section 4, edge metric dimension of prism related graph $\mathrm{D}_{\mathfrak{n}}^{\mathrm{t}}$ will be determined. In last Section, article will be concluded.

\section{Edge metric dimension of flower graph}

In this Section, we will investigate the edge metric dimension of flower graph $f_{n \times 3}$. We have the vertex set $V\left(f_{n \times 3}\right)=\left\{a_{\alpha}, b_{\alpha} \mid 1 \leqslant \alpha \leqslant n\right\}$ and the edge set $E\left(f_{n \times 3}\right)=\left\{a_{\alpha} a_{\alpha+1}, a_{\alpha} b_{\alpha}, a_{\alpha} b_{\alpha+1} \mid 1 \leqslant \alpha \leqslant n\right\}$ as shown in Figure 1. 


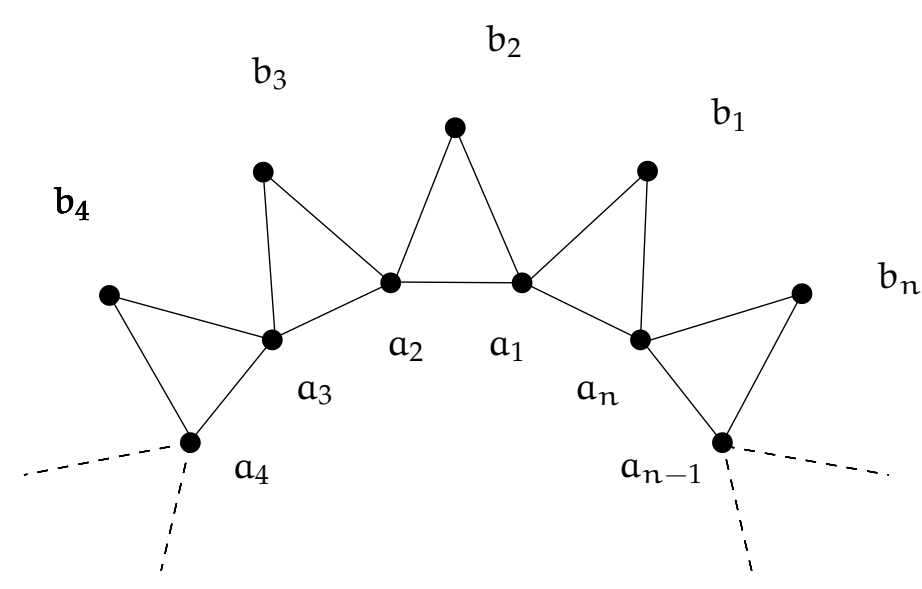

Figure 1: Graph of $f_{n \times 3}$.

Lemma 2.1. Let $\mathrm{Y}=\left\{\mathrm{b}_{1}, \mathrm{~b}_{2}, \ldots, \mathrm{b}_{\mathrm{n}}\right\}$ be a subset of $\mathrm{V}\left(\mathrm{f}_{\mathrm{n} \times 3}\right)$. Then any arbitrary edge metric generator $\mathrm{W}_{\mathrm{E}}$ of $f_{n \times 3}$ contains at least $\left\lceil\frac{\mathrm{n}}{2}\right\rceil$ vertices of $Y$.

Proof. Suppose that $W_{E}$ contains at most $\left\lceil\frac{n}{2}\right\rceil-1$ vertices of $Y$ for a contradiction. Without loss of generality we assume that $b_{\alpha}, b_{\alpha+1} \notin W E$, then we have $\left(a_{\alpha} b_{\alpha} \mid W\right)=\left(a_{\alpha} b_{\alpha+1} \mid W\right)$, a contradiction.

Remark 2.2. Let $W_{E}$ be any edge metric basis of $f_{n \times 3}$. We note that $W_{E}$ contains all odd vertices (vertex indices are odd) of $Y$ for odd $n$, while $W_{E}$ contains either all odd vertices or even vertices (vertex indices are even) of $Y$ for even $n$.

Theorem 2.3. For the flower graph $\mathrm{f}_{\mathrm{n} \times 3}$, we have

$$
\operatorname{edim}\left(f_{n \times 3}\right)= \begin{cases}3, & n=4 \\ 4, & n=3,5 \\ \left\lceil\frac{n}{2}\right\rceil, & \text { otherwise. }\end{cases}
$$

Proof. For $n=3,4,5$ we have calculated the edge metric dimension by total enumeration, $\operatorname{edim}\left(f_{3 \times 3}\right)=4$ and its edge metric basis is $\left\{a_{1}, a_{2}, b_{1}, b_{2}\right\}$, edim $\left(f_{4 \times 3}\right)=3$ and its edge metric basis is $\left\{b_{1}, b_{3}, b_{4}\right\}$, and $\operatorname{edim}\left(f_{5 \times 3}\right)=4$ and its edge metric basis is $\left\{a_{1}, b_{1}, b_{3}, b_{5}\right\}$.

For $n \geqslant 6$, we discuss the following four cases.

Let $W_{E}=\left\{b_{1}, b_{3}, b_{5}, \ldots, b_{n-1}\right\}$. We will show that $W_{E}$ is an edge metric basis of $f_{n \times 3}$ in Case(I) and Case(II), respectively.

Case (I) When $n \equiv 0(\bmod 4)$. We can write $n=2 k, k \geqslant 4$, and $k$ is even. Let $W_{1}=\left\{b_{1}, b_{3}, b_{k+1}, b_{k+3}\right\}$. Next, we give representation of edges of $f_{n \times 3}$ with respect to $W_{1}$.

$$
r\left(a_{\alpha} a_{\alpha+1} \mid W_{1}\right)= \begin{cases}(\alpha, 1, k-\alpha, \alpha+k-2), & 1 \leqslant \alpha \leqslant 2, \\ (\alpha, \alpha-2, k-\alpha, k-\alpha+2), & 3 \leqslant \alpha \leqslant k-1, \\ (k, k-2,1,2), & \alpha=k, \\ (n-\alpha, \alpha-2, \alpha-k, 1), & k+1 \leqslant \alpha \leqslant k+2, \\ (n-\alpha, n-\alpha+2, \alpha-k, \alpha-k-2), & k+3 \leqslant \alpha \leqslant n-1, \\ (1,2, k, k-2), & \alpha=n,\end{cases}
$$




$$
r\left(a_{\alpha} b_{\alpha} \mid W_{1}\right)= \begin{cases}(0,2, k, k-1), & \alpha=1, \\ (\alpha, 3-\alpha, k-\alpha+1, k), & 2 \leqslant \alpha \leqslant 3, \\ (\alpha, \alpha-2, k-\alpha+1, k-\alpha+3), & 4 \leqslant \alpha \leqslant k, \\ (n-k, k-1,0,2), & \alpha=k+1, \\ (n-k-1, k, 2,1), & \alpha=k+2, \\ (n-k-2, n-k, 3,0), & \alpha=k+3, \\ (n-\alpha+1, n-\alpha+3, \alpha-k, \alpha-k-2), & k+4 \leqslant \alpha \leqslant n, \\ r\left(a_{\alpha} b_{\alpha+1} \mid W_{1}\right)= \begin{cases}(1,2, k, k-1), & \alpha=1, \\ (2,0, k-1, k), & \alpha=2, \\ (\alpha, \alpha-2, k-\alpha+1, k-\alpha+3), & 3 \leqslant \alpha \leqslant k-1, \\ (n-k, k-1,0,3), & \alpha=k, \\ (n-k-1, k, 2,0), & \alpha=k+1, \\ (n-\alpha+1, n-\alpha+3, \alpha-k, \alpha-k-2), & \alpha+3 \leqslant \alpha \leqslant n-1, \\ (0,3, k, k-2), & \alpha=n .\end{cases} \end{cases}
$$

From above representation we see that $r\left(a_{\alpha} b_{\alpha} \mid W_{1}\right)=r\left(a_{\alpha} b_{\alpha+1} \mid W_{1}\right)$ when $1 \leqslant \alpha \leqslant n$ and $\alpha \neq 1,2,3, k, k+$ $1, k+2, k+3, n$ and no other edges have same representation. If we take odd $\alpha$, where $1 \leqslant \alpha \leqslant n$ and $\alpha \neq 1,3, k+1, n-1$ such that $W_{E}=W_{1} \cup\left\{b_{\alpha}\right\}$ then $r\left(a_{\alpha} b_{\alpha} \mid W_{E}\right) \neq r\left(a_{\alpha} b_{\alpha+1} \mid W_{E}\right)$ which implies that $\operatorname{edim}\left(f_{n \times 3}\right) \leqslant\left\lceil\frac{n}{2}\right\rceil$. So from Lemma 2.1, $W_{E}$ is an edge metric generator for $f_{n \times 3}$ and $\operatorname{edim}\left(f_{n \times 3}\right)=\left\lceil\frac{n}{2}\right\rceil$.

Case (II) When $n \equiv 2(\bmod 4)$. We can write $n=2 k, k \geqslant 3$, and $k$ is odd. Let $W_{1}=\left\{b_{1}, b_{3}, b_{k+2}\right\}$. Next, we give representation of edges of $f_{n \times 3}$ with respect to $W_{1}$.

$$
\begin{aligned}
& r\left(a_{\alpha} a_{\alpha+1} \mid W_{1}\right)= \begin{array}{ll}
(\alpha, 1, k-\alpha+1), & 1 \leqslant \alpha \leqslant 2, \\
(\alpha, \alpha-2, k-\alpha+1), & 3 \leqslant \alpha \leqslant k, \\
(n-k-1, k-1,1), & \alpha=k+1, \\
(n-\alpha, n-\alpha+2, \alpha-k-1), & k+2 \leqslant \alpha \leqslant n-1, \\
(1,2, n-k-1), & \alpha=n,
\end{array} \\
& r\left(a_{\alpha} b_{\alpha} \mid W_{1}\right)= \begin{cases}(0,2, k), & \alpha=1, \\
(\alpha, 3-\alpha, k-\alpha+2), & 2 \leqslant \alpha \leqslant 3, \\
(\alpha, \alpha-2, k-\alpha+2), & 4 \leqslant \alpha \leqslant k, \\
(n-\alpha+1, \alpha-2, k-\alpha+2), & k+1 \leqslant \alpha \leqslant k+2, \\
(n-\alpha+1, n-\alpha+3, \alpha-k-1), & k+3 \leqslant \alpha \leqslant n,\end{cases} \\
& r\left(a_{\alpha} b_{\alpha+1} \mid W_{1}\right)= \begin{cases}(1,2, k), & \alpha=1, \\
(\alpha, \alpha-2, k-\alpha+2), & 2 \leqslant \alpha \leqslant k, \\
(n-\alpha+1, \alpha-2, \alpha-k-1), & k+1 \leqslant \alpha \leqslant k+2, \\
(0,3, k-1), & k+3 \leqslant \alpha \leqslant n-1,\end{cases}
\end{aligned}
$$

From above representation we see that $r\left(a_{\alpha} b_{\alpha} \mid W_{1}\right)=r\left(a_{\alpha} b_{\alpha+1} \mid W_{1}\right)$ when $1 \leqslant \alpha \leqslant n$ and $\alpha \neq 1,2,3, k+$ $1, k+2, n$ and no other edges have same representation. If we take odd $\alpha$, where $1 \leqslant \alpha \leqslant n$ and $\alpha \neq$ $1,3, k+2$ such that $W_{E}=W_{1} \cup\left\{b_{\alpha}\right\}$ then $r\left(a_{\alpha} b_{\alpha} \mid W_{E}\right) \neq r\left(a_{\alpha} b_{\alpha+1} \mid W_{E}\right)$ which implies that edim $\left(f_{n \times 3}\right) \leqslant$ $\left\lceil\frac{n}{2}\right\rceil$. So from Lemma 2.1, $W_{E}$ is an edge metric generator for $f_{n \times 3}$ and edim $\left(f_{n \times 3}\right)=\left\lceil\frac{n}{2}\right\rceil$. 
Let $W_{E}=\left\{b_{1}, b_{3}, b_{5}, \ldots, b_{n-2}, b_{n}\right\}$. We will show that $W_{E}$ is an edge metric basis of $f_{n \times 3}$ in Cases (III) and (IV), respectively.

Case (III) When $n \equiv 1(\bmod 4)$. We can write $n=2 k+1, k \geqslant 4$, and $k$ is even. Let $W_{1}=\left\{b_{1}, b_{3}, b_{k+1}\right.$, $\left.b_{k+3}, b_{k+5}\right\}$. Next, we give representation of edges of $f_{n \times 3}$ with respect to $W_{1}$.

$$
\begin{aligned}
& r\left(a_{\alpha} a_{\alpha+1} \mid W_{1}\right)= \begin{cases}(1,1, k-1, k, k-2), & \alpha=1, \\
(\alpha, 1, k-\alpha, k-\alpha+2, \alpha+k-3), & 2 \leqslant \alpha \leqslant 3, \\
(\alpha, \alpha-2, k-\alpha, k-\alpha+2, k-\alpha+4), & 4 \leqslant \alpha \leqslant k-1, \\
(k, k-2,1,2,4), & \alpha=k, \\
(n-\alpha, \alpha-2, \alpha-k, 1, k-\alpha+4), & k+1 \leqslant \alpha \leqslant k+2, \\
(n-\alpha, n-\alpha+2, \alpha-k, \alpha-k-2,1), & k+3 \leqslant \alpha \leqslant k+4, \\
(n-\alpha, n-\alpha+2, \alpha-k, \alpha-k-2, \alpha-k-4), & k+5 \leqslant \alpha \leqslant n-1, \\
(1,2, k, k-1, k-3), & \alpha=n,\end{cases} \\
& r\left(a_{\alpha} b_{\alpha} \mid W_{1}\right)= \begin{cases}(0,2, k, k, k-2), & \alpha=1, \\
(\alpha, 3-\alpha, k-\alpha+1, k-\alpha+3, \alpha+k-3), & 2 \leqslant \alpha \leqslant 3, \\
(\alpha, \alpha-2, k-\alpha+1, k-\alpha+3, k-\alpha+5), & 4 \leqslant \alpha \leqslant k+1, \\
(n-\alpha+1, \alpha-2, \alpha-k, k-\alpha+3, k-\alpha+5), & k+2 \leqslant \alpha \leqslant k+3, \\
(n-\alpha+1, n-\alpha+3, \alpha-k, \alpha-k-2, k-\alpha+5), & k+4 \leqslant \alpha \leqslant k+5, \\
(n-\alpha+1, n-\alpha+3, \alpha-k, \alpha-k-2, \alpha-k-4), & k+6 \leqslant \alpha \leqslant n,\end{cases} \\
& r\left(a_{\alpha} b_{\alpha+1} \mid W_{1}\right)= \begin{cases}(1,2, k, k, k-2), & \alpha=1, \\
(\alpha, \alpha-2, k-\alpha+1, k-\alpha+3, \alpha+k-3), & 2 \leqslant \alpha \leqslant 3, \\
(\alpha, \alpha-2, k-\alpha+1, k-\alpha+3, k-\alpha+5), & 4 \leqslant \alpha \leqslant k-1, \\
(\alpha, \alpha-2, \alpha-k, k-\alpha+3, k-\alpha+5), & k \leqslant \alpha \leqslant k+1, \\
(n-\alpha+1, \alpha-2, \alpha-k, \alpha-k-2, k-\alpha+5), & k+2 \leqslant \alpha \leqslant k+3, \\
(n-\alpha+1, n-\alpha+3, \alpha-k, \alpha-k-2, \alpha-k-4), & k+4 \leqslant \alpha \leqslant n-1, \\
(0,3, k+1, k-1, k-3), & \alpha=n .\end{cases}
\end{aligned}
$$

From above representation we see that $r\left(a_{\alpha} b_{\alpha} \mid W_{1}\right)=r\left(a_{\alpha} b_{\alpha+1} \mid W_{1}\right)$ when $1 \leqslant \alpha \leqslant n$ and $\alpha \neq 1,2,3, k, k+$ $1, k+2, k+3, k+4, k+5, n$ and no other edges have same representation. If we take odd $\alpha$, where $1 \leqslant \alpha \leqslant n$ and $\alpha \neq 1,3, k+1, k+3, n-1$ such that $W_{E}=W_{1} \cup\left\{b_{\alpha}\right\}$ then $r\left(a_{\alpha} b_{\alpha} \mid W_{E}\right) \neq r\left(a_{\alpha} b_{\alpha+1} \mid W_{E}\right)$ which implies that edim $\left(f_{n \times 3}\right) \leqslant\left\lceil\frac{n}{2}\right\rceil$. So from Lemma $2.1, W_{E}$ is an edge metric generator for $f_{n \times 3}$ and $\operatorname{edim}\left(f_{n \times 3}\right)=\left\lceil\frac{\mathfrak{n}}{2}\right\rceil$.

Case (IV) When $n \equiv 3(\bmod 4)$. We can write $n=2 k+1, k \geqslant 3, k$ is odd. Let $W_{1}=\left\{b_{1}, b_{3}, b_{k+2}, b_{k+4}\right\}$. Next, we give representation of edges of $f_{n \times 3}$ with respect to $W_{1}$.

$$
r\left(a_{\alpha} a_{\alpha+1} \mid W_{1}\right)= \begin{cases}(\alpha, 1, k-\alpha+1, \alpha+k-2), & 1 \leqslant \alpha \leqslant 2, \\ (\alpha, \alpha-2, k-\alpha+1, k-\alpha+3), & 3 \leqslant \alpha \leqslant k-1, \\ (k, \alpha-2,1, k-\alpha+3), & k \leqslant \alpha \leqslant k+1, \\ (n-\alpha, k, \alpha-k-1,1), & k+2 \leqslant \alpha \leqslant k+3, \\ (n-\alpha, n-\alpha+2, \alpha-k-1, \alpha-k-3), & k+4 \leqslant \alpha \leqslant n-1, \\ (1,2, n-k-1, n-k-3), & \alpha=n,\end{cases}
$$




$$
\begin{aligned}
r\left(a_{\alpha} b_{\alpha} \mid W_{1}\right)= \begin{cases}(0,2, k+1, n-k-2), & \alpha=1, \\
(\alpha, 3-\alpha, k-\alpha+2, n-k+\alpha-3), & 2 \leqslant \alpha \leqslant 3, \\
(\alpha, \alpha-2, k-\alpha+2, k-\alpha+4), & 4 \leqslant \alpha \leqslant k+1, \\
(n-k-1, k, 0,2), & \alpha=k+2, \\
(n-\alpha+1, n-\alpha+3, \alpha-k-1, k-\alpha+4), & k+3 \leqslant \alpha \leqslant k+4, \\
(n-\alpha+1, n-\alpha+3, \alpha-k-1, \alpha-k-3), & k+5 \leqslant \alpha \leqslant n,\end{cases} \\
r\left(a_{\alpha} b_{\alpha+1} \mid W_{1}\right)= \begin{cases}(1,2, k+1, n-k-2), & \alpha=1, \\
(2,0, k, n-k-1), & \alpha=2, \\
(\alpha, \alpha-2, k-\alpha+2, k-\alpha+4), & 3 \leqslant \alpha \leqslant k, \\
(n-\alpha+1, \alpha-2, \alpha-k-1, k-\alpha+4), & k+1 \leqslant \alpha \leqslant k+2, \\
(0,3, n-k-1, n-k-3), & k+3 \leqslant \alpha \leqslant n-1,\end{cases}
\end{aligned}
$$

From above representation we see that $r\left(a_{\alpha} b_{\alpha} \mid W_{1}\right)=r\left(a_{\alpha} b_{\alpha+1} \mid W_{1}\right)$ when $1 \leqslant \alpha \leqslant n$ and $\alpha \neq 1,2,3, k+$ $1, k+2, k+3, k+4, n$ and no other edges have same representation. If we take odd $\alpha$, where $1 \leqslant \alpha \leqslant n$ and $\alpha \neq 1,3, k+2, k+4$ such that $W_{E}=W_{1} \cup\left\{b_{\alpha}\right\}$ then $r\left(a_{\alpha} b_{\alpha} \mid W_{E}\right) \neq r\left(a_{\alpha} b_{\alpha+1} \mid W_{E}\right)$ which implies that edim $\left(f_{n \times 3}\right) \leqslant\left\lceil\frac{n}{2}\right\rceil$. So from Lemma 2.1, $W_{E}$ is an edge metric generator for $f_{n \times 3}$ and $\operatorname{edim}\left(f_{n \times 3}\right)=$ $\left\lceil\frac{\mathrm{n}}{2}\right\rceil$.

\section{Edge metric dimension of $D_{n}^{\prime}$}

The prism related graph $D_{n}^{\prime}$ has vertex set $V\left(D_{n}^{\prime}\right)=\left\{a_{\alpha}, b_{\alpha}, c_{\alpha}, d_{\alpha}, e_{\alpha} \mid 1 \leqslant \alpha \leqslant n\right\}$ and the edge set $E\left(D_{n}^{\prime}\right)=\left\{a_{\alpha} a_{\alpha+1}, a_{\alpha} b_{\alpha}, c_{\alpha} b_{\alpha}, c_{\alpha} b_{\alpha+1}, c_{\alpha} c_{\alpha+1}, c_{\alpha} d_{\alpha}, d_{\alpha} d_{\alpha+1}, d_{\alpha} e_{\alpha} \mid 1 \leqslant \alpha \leqslant n\right\}$ as shown in Figure 2. In this Section, we determine the edge metric of the graph $D_{n}^{\prime}$.

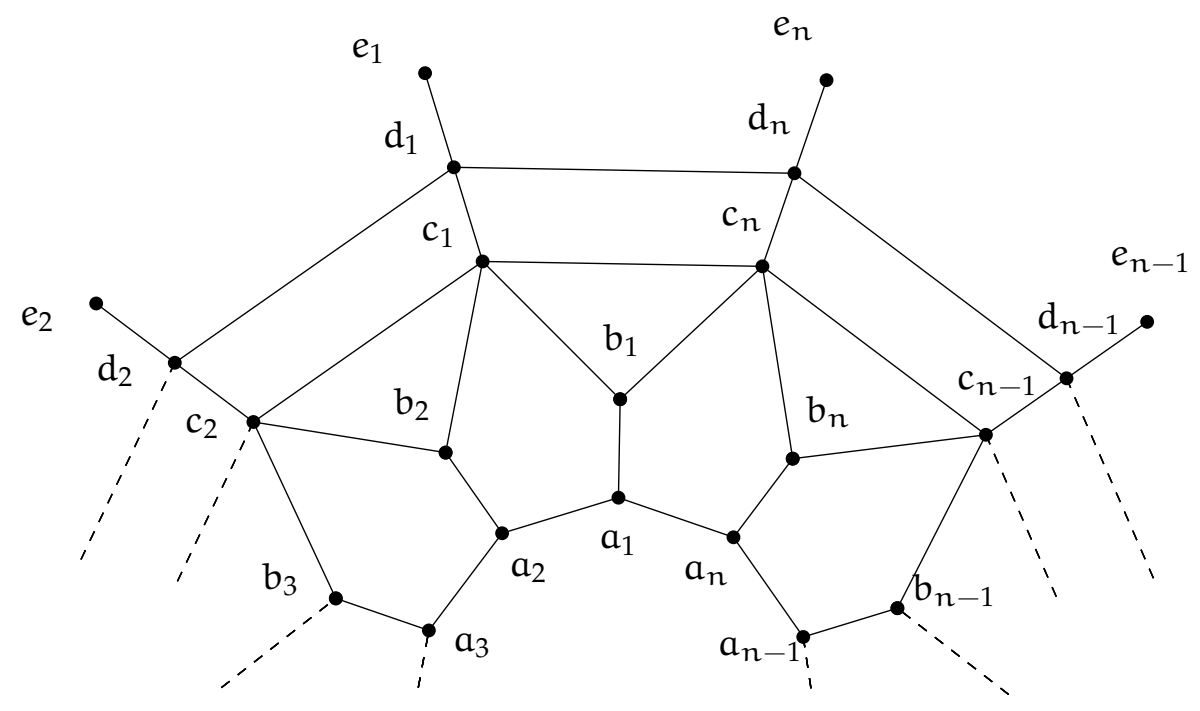

Figure 2: Graph of $D_{n}^{\prime}$.

Theorem 3.1. For the graph $\mathrm{D}_{\mathrm{n}}^{\prime}$ with $\mathrm{n} \geqslant 4$, we have

$$
\operatorname{edim}\left(D_{n}^{\prime}\right)= \begin{cases}3, & n \text { is even and } n \neq 4 \\ 4, & n \text { is odd and } n=4\end{cases}
$$


Proof. For $\mathrm{n}=4$, we have calculated the edge metric dimension by total enumeration, $\operatorname{edim}\left(\mathrm{D}_{4}^{\prime}\right)=4$ and its edge metric basis is $\left\{a_{1}, a_{2}, b_{3}, b_{4}\right\}$.

For $n>4$, we discuss the two cases.

Case (i) When $n$ is even. We can write $n=2 k$, for $k \geqslant 3$. Let $W_{E}=\left\{a_{1}, e_{k-1}, e_{n-1}\right\}$ is an edge metric basis for $D_{n}^{\prime}$. We give representations of any edge of $E\left(D_{n}^{\prime}\right)$ with respect to $W_{E}$.

$$
\begin{aligned}
& r\left(a_{\alpha} a_{\alpha+1} \mid W_{E}\right)= \begin{cases}(\alpha-1, k-\alpha+2, \alpha+4), & 1 \leqslant \alpha \leqslant k-2, \\
(\alpha-1,4, n-\alpha+2), & k-1 \leqslant \alpha \leqslant k, \\
(n-\alpha, \alpha-k+4, n-\alpha+2), & k+1 \leqslant \alpha \leqslant n-2, \\
(n-\alpha, n-\alpha+k+2,4), & n-1 \leqslant \alpha \leqslant n,\end{cases} \\
& r\left(a_{\alpha} b_{\alpha} \mid W_{E}\right)= \begin{cases}(\alpha-1, k-\alpha+2, \alpha+3), & 1 \leqslant \alpha \leqslant k-1, \\
(k-1,3, n-k+2), & \alpha=k, \\
(n-\alpha+1, \alpha-k+3, n-\alpha+2), & k+1 \leqslant \alpha \leqslant n-1, \\
(1, k+2,3), & \alpha=n,\end{cases} \\
& r\left(b_{\alpha} c_{\alpha} \mid W_{E}\right)= \begin{cases}(\alpha, k-\alpha+1, \alpha+3), & 1 \leqslant \alpha \leqslant k-1, \\
(k, 3, n-k+1), & \alpha=k, \\
(n-\alpha+2, \alpha-k+3, n-\alpha+1), & k+1 \leqslant \alpha \leqslant n-1, \\
(2, k+1,3), & \alpha=n,\end{cases} \\
& r\left(c_{\alpha} b_{\alpha+1} \mid W_{E}\right)= \begin{cases}(\alpha+1, k-\alpha+1, \alpha+3), & 1 \leqslant \alpha \leqslant k-1, \\
(k+1,3, n-k+1), & \alpha=k, \\
(n-\alpha+1, \alpha-k+3, n-\alpha+1), & k+1 \leqslant \alpha \leqslant n-1, \\
(1, k+1,3), & \alpha=n,\end{cases} \\
& r\left(c_{\alpha} c_{\alpha+1} \mid W_{E}\right)= \begin{cases}(\alpha+1, k-\alpha, \alpha+3), & 1 \leqslant \alpha \leqslant k-2, \\
(\alpha+1, \alpha-k+3, n-\alpha), & k-1 \leqslant \alpha \leqslant k \\
(n-\alpha+1, \alpha-k+3, n-\alpha), & k+1 \leqslant \alpha \leqslant n-2 \\
(2, n-\alpha+k, \alpha-n+3), & n-1 \leqslant \alpha \leqslant n\end{cases} \\
& r\left(c_{\alpha} d_{\alpha} \mid W_{E}\right)= \begin{cases}(\alpha+1, k-\alpha, \alpha+2), & 1 \leqslant \alpha \leqslant k-1, \\
(k+1,2, n-k), & \alpha=k, \\
(n-\alpha+2, \alpha-k+2, n-\alpha), & k+1 \leqslant \alpha \leqslant n-1, \\
(2, k, 2), & \alpha=n,\end{cases} \\
& r\left(d_{\alpha} e_{\alpha} \mid W_{E}\right)= \begin{cases}(\alpha+2, k-\alpha, \alpha+2), & 1 \leqslant \alpha \leqslant k-2, \\
(k+1,0, k+1), & \alpha=k-1, \\
(k+2,2, n-k), & \alpha=k, \\
(n-\alpha+3, \alpha-k+2, n-\alpha), & k+1 \leqslant \alpha \leqslant n-2, \\
(4, n-k+1,0), & \alpha=n-1, \\
(3, k, 2), & \alpha=n,\end{cases} \\
& r\left(d_{\alpha} d_{\alpha+1} \mid W_{E}\right)= \begin{cases}(\alpha+2, k-\alpha-1, \alpha+2), & 1 \leqslant \alpha \leqslant k-2, \\
(\alpha+2, \alpha-k+2, n-\alpha-1), & k-1 \leqslant \alpha \leqslant k, \\
(n-\alpha+2, \alpha-k+2, n-\alpha-1), & k+1 \leqslant \alpha \leqslant n-2, \\
(3, n-\alpha+k-1, \alpha-n+2), & n-1 \leqslant \alpha \leqslant n .\end{cases}
\end{aligned}
$$


We note that there are no vertices having the same edge metric representation implying that edim $\left(D_{n}{ }^{\prime}\right) \leqslant$ 3. Using Proposition 1.1, edim $\left(D_{n}{ }^{\prime}\right) \geqslant 3$, which implies edim $\left(D_{n}{ }^{\prime}\right)=3$.

Case (ii) When $n$ is odd. We can write $n=2 k+1$, for $k \geqslant 2$. Let $W_{E}=\left\{a_{1}, a_{k+2}, b_{2}, b_{k+1}\right\}$ is an edge metric basis for $D_{n}^{\prime}$. We give representations of any edge with respect to $W_{E}$.

$$
\begin{aligned}
& r\left(a_{\alpha} a_{\alpha+1} \mid W_{E}\right)= \begin{cases}(0, k, 1, k), & \alpha=1, \\
(\alpha-1, k-\alpha+1, \alpha-1, k-\alpha+1), & 2 \leqslant \alpha \leqslant k, \\
(n-k-1,0, k, 1), & \alpha=k+1, \\
(n-\alpha, \alpha-k-2, n-\alpha+2, \alpha-k), & k+2 \leqslant \alpha \leqslant n,\end{cases} \\
& r\left(a_{\alpha} b_{\alpha} \mid W_{E}\right)= \begin{cases}(0, k, 2, k+1), & \alpha=1, \\
(1, k, 0, k), & \alpha=2, \\
(\alpha-1, k-\alpha+2, \alpha-1, k-\alpha+2), & 3 \leqslant \alpha \leqslant k, \\
(k, 1, k, 0), & \alpha=k+1, \\
(n-k-1,0, k+1,2), & \alpha=k+2, \\
(n-\alpha+1, \alpha-k-2, n-\alpha+3, \alpha-k), & k+3 \leqslant \alpha \leqslant n,\end{cases} \\
& r\left(b_{\alpha} c_{\alpha} \mid W_{E}\right)= \begin{cases}(1, k+1,1, k), & \alpha=1, \\
(2, k+1,0, k-1), & \alpha=2, \\
(\alpha, k-\alpha+3, \alpha-1, k-\alpha+1), & 3 \leqslant \alpha \leqslant k+1, \\
(n-\alpha+2, \alpha-k-1, n-\alpha+2, \alpha-k), & k+2 \leqslant \alpha \leqslant n,\end{cases} \\
& r\left(c_{\alpha} b_{\alpha+1} \mid W_{E}\right)= \begin{cases}(\alpha+1, k-\alpha+2, \alpha-1, k-\alpha+1), & 1 \leqslant \alpha \leqslant k-1, \\
(k+1,2, k-1,0), & \alpha=k, \\
(k+2,1, k, 1), & \alpha=k+1, \\
(n-\alpha+1, \alpha-k, n-\alpha+2, \alpha-k), & k+2 \leqslant \alpha \leqslant n,\end{cases} \\
& r\left(c_{\alpha} c_{\alpha+1} \mid W_{E}\right)= \begin{cases}(2, k+1,1, k-1), & \alpha=1, \\
(\alpha+1, k-\alpha+2, \alpha-1, k-\alpha), & 2 \leqslant \alpha \leqslant k-1, \\
(k+1,2, \alpha-1,1), & k \leqslant \alpha \leqslant k+1, \\
(n-\alpha+1, \alpha-k, n-\alpha+1, \alpha-k), & k+2 \leqslant \alpha \leqslant n-1, \\
(2, k+1,1, k), & \alpha=n,\end{cases} \\
& r\left(c_{\alpha} d_{\alpha} \mid W_{E}\right)= \begin{cases}(2, k+2,1, k), & \alpha=1, \\
(\alpha+1, k-\alpha+3, \alpha-1, k-\alpha+1), & 2 \leqslant \alpha \leqslant k, \\
(k+2,2, k, 1), & \alpha=k+1, \\
(n-\alpha+2, \alpha-k, n-\alpha+2, \alpha-k), & k+2 \leqslant \alpha \leqslant n,\end{cases} \\
& r\left(d_{\alpha} d_{\alpha+1} \mid W_{E}\right)= \begin{cases}(3, k+2,2, k), & \alpha=1, \\
(\alpha+2, k-\alpha+3, \alpha, k-\alpha+1), & 2 \leqslant \alpha \leqslant k-1, \\
(k+2,3, \alpha, 2), & k \leqslant \alpha \leqslant k+1, \\
(n-\alpha+2, \alpha-k+1, n-\alpha+2, \alpha-k+1), & k+2 \leqslant \alpha \leqslant n-1, \\
(3, k+2,2, k+1), & \alpha=n,\end{cases} \\
& r\left(d_{\alpha} e_{\alpha} \mid W_{E}\right)= \begin{cases}(3, k+3,2, k+1), & \alpha=1, \\
(\alpha+2, k-\alpha+4, \alpha, k-\alpha+2), & 2 \leqslant \alpha \leqslant k, \\
(k+3,3, k+1,2), & \alpha=k+1, \\
(n-\alpha+3, \alpha-k+1, n-\alpha+3, \alpha-k+1), & k+2 \leqslant \alpha \leqslant n .\end{cases}
\end{aligned}
$$

We note that there are no vertices having the same edge metric representation implying that edim $\left(D_{n}{ }^{\prime}\right) \leqslant$ 4.

Suppose on contrary that edim $\left(D_{n}{ }^{\prime}\right)=3$, then the Table 1 shows all order pairs of edges $(e, f)$ for which $r\left(e \mid W_{E}\right)=r\left(f \mid W_{E}\right)$. 
Table 1: $(e, f)$ for which $r\left(e \mid W_{E}\right)=r\left(f \mid W_{E}\right)$.

\begin{tabular}{|c|c|}
\hline$W_{E}$ & $(e, f)$ \\
\hline$\left\{a_{1}, a_{j}, a_{l}\right\}$ where $2 \leqslant j \leqslant k+1$ and $3 \leqslant l \leqslant n$ & $\begin{array}{l}\text { For } 3 \leqslant l \leqslant k+1, r\left(a_{1} a_{n} \mid W_{E}\right)=r\left(a_{1} b_{1} \mid W_{E}\right) \\
\text { And for } k+2 \leqslant l \leqslant n, r\left(b_{1} c_{1} \mid W_{E}\right)=r\left(b_{1} c_{n} \mid W_{E}\right)\end{array}$ \\
\hline$\left\{b_{1}, b_{j}, b_{l}\right\}$ where $2 \leqslant j \leqslant k+1$ and $3 \leqslant l \leqslant n$ & $\begin{array}{l}\text { For } 3 \leqslant l \leqslant k+1, r\left(d_{1} d_{n} \mid W_{E}\right)=r\left(d_{1} e_{1} \mid W_{E}\right) \\
\text { And for } k+2 \leqslant l \leqslant n, r\left(d_{1} d_{n} \mid W_{E}\right)=r\left(d_{n} e_{n} \mid W_{E}\right) .\end{array}$ \\
\hline$\left\{c_{1}, c_{j}, c_{l}\right\}$ where $2 \leqslant j \leqslant k+1$ and $3 \leqslant l \leqslant n$ & $\begin{array}{l}\text { For } 3 \leqslant l \leqslant k+1, r\left(d_{1} d_{n} \mid W_{E}\right)=r\left(d_{1} e_{1} \mid W_{E}\right) \\
\text { And for } k+2 \leqslant l \leqslant n, r\left(d_{1} d_{2} \mid W_{E}\right)=r\left(d_{1} e_{1} \mid W_{E}\right)\end{array}$ \\
\hline$\left\{d_{1}, d_{j}, d_{l}\right\}$ where $2 \leqslant j \leqslant k+1$ and $3 \leqslant l \leqslant n$ & This case is same to case (3) due to symmetry of the graph. \\
\hline$\left\{e_{1}, e_{j}, e_{l}\right\}$ where $2 \leqslant j \leqslant k+1$ and $3 \leqslant l \leqslant n$ & $\begin{array}{l}\text { For } 3 \leqslant l \leqslant k+1, r\left(d_{1} d_{n} \mid W_{E}\right)=r\left(c_{1} d_{1} \mid W_{E}\right) \\
\text { And for } k+2 \leqslant l \leqslant n, r\left(d_{1} d_{2} \mid W_{E}\right)=r\left(c_{1} d_{1} \mid W_{E}\right)\end{array}$ \\
\hline$\left\{a_{1}, a_{j}, b_{l}\right\}$ where $2 \leqslant j \leqslant k+1$ and $1 \leqslant l \leqslant n$ & $\begin{array}{l}\text { For } 1 \leqslant l \leqslant k+1, r\left(e_{k} d_{k} \mid W_{E}\right)=r\left(d_{k} d_{k+1} \mid W_{E}\right) \\
\text { For } k+2 \leqslant l \leqslant n-1, r\left(b_{2} c_{2} \mid W_{E}\right)=r\left(c_{1} b_{2} \mid W_{E}\right) \\
\text { And for } l=n, r\left(c_{k+1} d_{k+1} \mid W_{E}\right)=r\left(d_{k} d_{k+1} \mid W_{E}\right)\end{array}$ \\
\hline$\left\{a_{1}, a_{j}, c_{l}\right\}$ where $2 \leqslant j \leqslant k+1$ and $1 \leqslant l \leqslant n$ & $\begin{array}{l}\text { For } 1 \leqslant l \leqslant k+1, r\left(d_{1} d_{n} \mid W_{E}\right)=r\left(d_{1} e_{1} \mid W_{E}\right) \\
\text { And for } k+2 \leqslant l \leqslant n, r\left(d_{1} d_{2} \mid W_{E}\right)=r\left(d_{1} e_{1} \mid W_{E}\right)\end{array}$ \\
\hline$\left\{a_{1}, a_{j}, e_{l}\right\}$ where $2 \leqslant j \leqslant k+1$ and $1 \leqslant l \leqslant n$ & $\begin{array}{l}\text { For } 1 \leqslant l \leqslant k, r\left(d_{n-1} d_{n} \mid W_{E}\right)=r\left(d_{n} e_{n} \mid W_{E}\right) \\
\text { For } l=k+1, r\left(d_{1} d_{n} \mid W_{E}\right)=r\left(d_{1} e_{1} \mid W_{E}\right) \\
\text { And for } k+2 \leqslant l \leqslant n, r\left(d_{1} d_{2} \mid W_{E}\right)=r\left(d_{1} e_{1} \mid W_{E}\right)\end{array}$ \\
\hline$\left\{a_{1}, b_{j}, b_{l}\right\}$ where $1 \leqslant j \leqslant k+1$ and $2 \leqslant l \leqslant n$ & $\begin{array}{l}\text { For } 2 \leqslant l \leqslant k, r\left(d_{1} d_{n} \mid W_{E}\right)=r\left(d_{1} e_{1} \mid W_{E}\right) \\
\text { For } k+2 \leqslant l \leqslant n-1, r\left(c_{1} b_{2} \mid W_{E}\right)=r\left(b_{2} c_{2} \mid W_{E}\right) \\
\text { And for } l=n, r\left(d_{1} d_{2} \mid W_{E}\right)=r\left(d_{1} e_{1} \mid W_{E}\right)\end{array}$ \\
\hline$\left\{a_{1}, b_{j}, c_{l}\right\}$ where $1 \leqslant j \leqslant k+1$ and $1 \leqslant l \leqslant n$ & $\begin{array}{l}\text { For } 1 \leqslant l \leqslant k+1, r\left(d_{1} d_{n} \mid W_{E}\right)=r\left(d_{1} e_{1} \mid W_{E}\right) \\
\text { And for } k+2 \leqslant l \leqslant n, r\left(d_{1} d_{2} \mid W_{E}\right)=r\left(d_{1} e_{1} \mid W_{E}\right)\end{array}$ \\
\hline$\left\{a_{1}, b_{j}, e_{l}\right\}$ where $1 \leqslant j \leqslant k+1$ and $1 \leqslant l \leqslant n$ & $\begin{array}{l}\text { For } 1 \leqslant l \leqslant k, r\left(d_{n-1} d_{n} \mid W_{E}\right)=r\left(d_{n} e_{n} \mid W_{E}\right) \\
\text { For } l=k+1, r\left(d_{1} d_{n} \mid W_{E}\right)=r\left(d_{1} e_{1} \mid W_{E}\right) \\
\text { And for } k+2 \leqslant l \leqslant n, r\left(d_{1} d_{2} \mid W_{E}\right)=r\left(d_{1} e_{1} \mid W_{E}\right)\end{array}$ \\
\hline$\left\{a_{1}, c_{j}, c_{l}\right\}$ where $1 \leqslant j \leqslant k+1$ and $2 \leqslant l \leqslant n$ & $\begin{array}{l}\text { For } 2 \leqslant l \leqslant k+1, r\left(d_{1} d_{n} \mid W_{E}\right)=r\left(d_{1} e_{1} \mid W_{E}\right) \\
\text { And for } k+2 \leqslant l \leqslant n, r\left(d_{1} d_{2} \mid W_{E}\right)=r\left(d_{1} e_{1} \mid W_{E}\right)\end{array}$ \\
\hline$\left\{a_{1}, c_{j}, d_{l}\right\}$ where $1 \leqslant j \leqslant k+1$ and $1 \leqslant l \leqslant n$ & $\begin{array}{l}\text { For } 1 \leqslant l \leqslant k+1, r\left(d_{1} d_{n} \mid W_{E}\right)=r\left(d_{1} e_{1} \mid W_{E}\right) \\
\text { And for } k+2 \leqslant l \leqslant n, r\left(d_{1} d_{2} \mid W_{E}\right)=r\left(d_{1} e_{1} \mid W_{E}\right)\end{array}$ \\
\hline$\left\{a_{1}, e_{j}, e_{l}\right\}$ where $1 \leqslant j \leqslant k+1$ and $2 \leqslant l \leqslant n$ & $\begin{array}{l}\text { For } 2 \leqslant l \leqslant k, r\left(d_{n} d_{n-1} \mid W_{E}\right)=r\left(d_{n} e_{n} \mid W_{E}\right) . \\
\text { For } l=k+1, r\left(d_{n-1} d_{n} \mid W_{E}\right)=r\left(c_{2} d_{2} \mid W_{E}\right) . \\
\text { And for } k+2 \leqslant l \leqslant n, r\left(d_{k+1} c_{k+1} \mid W_{E}\right)=r\left(c_{k+1} b_{k+2} \mid W_{E}\right) .\end{array}$ \\
\hline$\left\{b_{1}, e_{j}, e_{l}\right\}$ where $1 \leqslant j \leqslant k+1$ and $2 \leqslant l \leqslant n$ & $\begin{array}{l}\text { For } 2 \leqslant l \leqslant k, r\left(d_{n} d_{n-1} \mid W_{E}\right)=r\left(d_{n} e_{n} \mid W_{E}\right) \\
\text { And for } k+1 \leqslant l \leqslant n, r\left(d_{2} e_{2} \mid W_{E}\right)=r\left(c_{2} d_{2} \mid W_{E}\right)\end{array}$ \\
\hline$\left\{d_{1}, d_{j}, e_{l}\right\}$ where $2 \leqslant j \leqslant k+1$ and $1 \leqslant l \leqslant n$ & $\begin{array}{l}\text { For } 1 \leqslant l \leqslant k+1, r\left(d_{1} d_{n} \mid W_{E}\right)=r\left(c_{1} d_{1} \mid W_{E}\right) \\
\text { And for } k+2 \leqslant l \leqslant n, r\left(d_{2} e_{2} \mid W_{E}\right)=r\left(c_{2} d_{2} \mid W_{E}\right)\end{array}$ \\
\hline$\left\{d_{1}, e_{j}, e_{l}\right\}$ where $1 \leqslant j \leqslant k+1$ and $2 \leqslant l \leqslant n$ & $\begin{array}{l}\text { For } 2 \leqslant l \leqslant k+1, r\left(d_{1} d_{n} \mid W_{E}\right)=r\left(c_{1} d_{1} \mid W_{E}\right) \\
\text { For } k+2 \leqslant l \leqslant n, r\left(d_{2} e_{2} \mid W_{E}\right)=r\left(c_{2} d_{2} \mid W_{E}\right)\end{array}$ \\
\hline
\end{tabular}

Hence, Table 1 follows that there is no resolving set with three vertices for $V\left(D_{n}{ }^{\prime}\right)$ implying that $\operatorname{edim}\left(D_{n}{ }^{\prime}\right)=4$. Which completes the proof.

\section{Edge metric dimension of $D_{n}^{t}$}

The prism related graph $D_{n}^{t}$ has vertex set $V\left(D_{n}^{t}\right)=\left\{a_{\alpha}, b_{\alpha}, c_{\alpha}, d_{\alpha}: 1 \leqslant \alpha \leqslant n\right\}$ and the edge set $E\left(D_{n}^{t}\right)=\left\{a_{\alpha} a_{\alpha+1}, a_{\alpha} b_{\alpha}, b_{\alpha} b_{\alpha+1}, b_{\alpha} c_{\alpha}, b_{\alpha} c_{\alpha+1}, c_{\alpha} d_{\alpha}: 1 \leqslant \alpha \leqslant n\right\}$ as shown in Figure 3. In this Section, we determine the edge metric dimension of the graph $D_{n}^{t}$.

Lemma 4.1. Let $\mathrm{Y}=\left\{\mathrm{d}_{1}, \mathrm{~d}_{2}, \ldots, \mathrm{d}_{\mathrm{n}}\right\}$ be a subset of $\mathrm{V}\left(\mathrm{D}_{\mathrm{n}}^{\mathrm{t}}\right)$. Then any arbitrary edge metric generator $\mathrm{W}_{\mathrm{E}}$ of $\mathrm{D}_{\mathrm{n}}^{\mathrm{t}}$ contains at least $\left\lceil\frac{\mathfrak{n}}{2}\right\rceil$ vertices. 


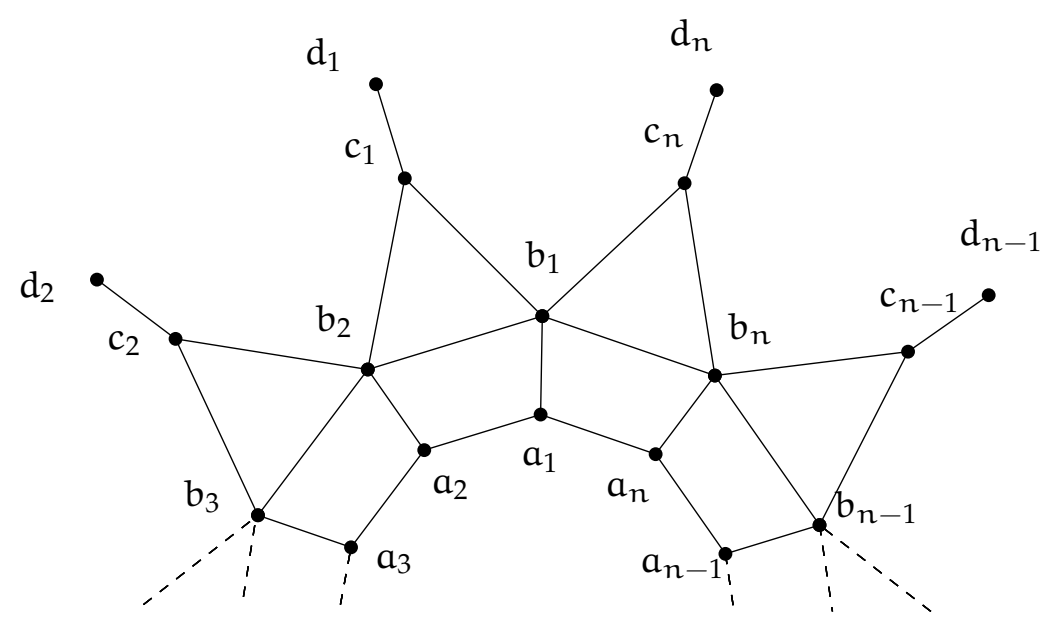

Figure 3: Graph of $D_{n}^{t}$.

Proof. Suppose that $W_{E}$ contains at most $\left\lceil\frac{n}{2}\right\rceil-1$ vertices of $Y$ for a contradiction. Without loss of generality we assume that $d_{\alpha}, d_{\alpha+1} \notin W E$, then we have $\left(b_{\alpha} c_{\alpha} \mid W_{E}\right)=\left(b_{\alpha} c_{\alpha+1} \mid W_{E}\right)$, a contradiction.

Remark 4.2. Let $W_{E}$ be any edge metric basis of $D_{n}^{t}$. We note that $W_{E}$ contains all odd vertices (vertex indices are odd) of $Y$ for odd $n$, while $W_{E}$ contains either all odd vertices or even vertices (vertex indices are even) of $Y$ for even $n$.

Lemma 4.3. For $n \geqslant 5$, we have edim $\left(D_{n}^{t}\right) \geqslant\left\lceil\frac{n}{2}\right\rceil+1$

Proof. We assume for a contradiction that the cardinality of subset $W_{E}$ is equal to $\left\lceil\frac{\mathrm{n}}{2}\right\rceil$ by Lemma 4.1. Using Remark 4.2, we take $W_{E}=\left\{d_{\alpha} \in W_{E} \mid\right.$ Vertices indices $\alpha$ is odd $\}$ such that $W_{E}=\left\lceil\frac{\mathfrak{n}}{2}\right\rceil$. We have $\left(a_{\alpha} b_{\alpha} \mid W_{E}\right)=\left(b_{\alpha} c_{\alpha} \mid W_{E}\right)$ for even $\alpha(1 \leqslant \alpha \leqslant n)$, a contradiction. So, edim $\left(D_{n}^{t}\right) \geqslant\left\lceil\frac{\mathfrak{n}}{2}\right\rceil+1$.

Theorem 4.4. For the graph $\mathrm{D}_{\mathrm{n}}^{\mathrm{t}}$ with $\mathrm{n} \geqslant 3$, we have

$$
\operatorname{edim}\left(D_{n}^{t}\right)= \begin{cases}4, & n=3,4 \\ \left\lceil\frac{n}{2}\right\rceil+1, & \text { otherwise. }\end{cases}
$$

Proof. For $n=3,4$ we have calculated the edge metric dimension by total enumeration that is 4 and its edge metric basis is $\left\{c_{1}, c_{2}, c_{3}, c_{4}\right\}$ and $\left\{a_{1}, b_{3}, c_{1}, c_{2}\right\}$, respectively.

For $n \geqslant 5$, we discuss the following four cases.

Let $W_{E}=\left\{a_{1}, d_{1}, d_{3}, d_{5}, \ldots, d_{n-1}\right\}$. We will show that $W_{E}$ is an edge metric basis of $D_{n}^{t}$ in Case(I) and Case(II), respectively.

Case (I) When $n \equiv 0(\bmod 4)$. We can write $n=2 k, k \geqslant 4$, and $k$ is even. Let $W_{1}=\left\{a_{1}, d_{1}, d_{3}, d_{k+1}, d_{k+3}\right\}$. Next, we give representation of edges of $D_{n}^{t}$ with respect to $W_{1}$.

$$
\begin{aligned}
r\left(a_{\alpha} a_{\alpha+1} \mid W_{1}\right) & = \begin{cases}(\alpha-1, \alpha+2,3, k-\alpha+2, \alpha+k), & 1 \leqslant \alpha \leqslant 2, \\
(\alpha-1, \alpha+2, \alpha, k-\alpha+2, k-\alpha+4), & 3 \leqslant \alpha \leqslant k-1, \\
(k-1, k+2, k, 3,4), & \alpha=k, \\
(n-\alpha, n-\alpha+2, \alpha, \alpha-k+2,3), & k+1 \leqslant \alpha \leqslant k+2, \\
(n-\alpha, n-\alpha+2, n-\alpha+4, \alpha-k+2, \alpha-k), & k+3 \leqslant \alpha \leqslant n-1, \\
(0,3,4, k+2, k), & \alpha=n,\end{cases} \\
r\left(a_{\alpha} b_{\alpha} \mid W_{1}\right) & = \begin{cases}(\alpha-1, \alpha+1,4-\alpha, k-\alpha+2, \alpha+k-1), & 1 \leqslant \alpha \leqslant 2, \\
(\alpha-1, \alpha+1, \alpha-1, k-\alpha+2, k-\alpha+4), & 3 \leqslant \alpha \leqslant k, \\
(n-\alpha+1, n-\alpha+2, \alpha-1, \alpha-k+1, k-\alpha+4), & k+1 \leqslant \alpha \leqslant k+2, \\
(n-\alpha+1, n-\alpha+2, n-\alpha+4, \alpha-k+1, \alpha-k-1), & k+3 \leqslant \alpha \leqslant n,\end{cases}
\end{aligned}
$$




$$
\begin{aligned}
& \left(\begin{array}{ll}
(\alpha, \alpha+1,2, k-\alpha+1, \alpha+k-1), & 1 \leqslant \alpha \leqslant 2, \\
(\alpha, \alpha+1, \alpha-1, k-\alpha+1, k-\alpha+3), & 3 \leqslant \alpha \leqslant k
\end{array}\right. \\
& r\left(b_{\alpha} b_{\alpha+1} \mid W_{1}\right)= \begin{cases}(\alpha, \alpha+1, \alpha-1, k-\alpha+1, k-\alpha+3), & 3 \leqslant \alpha \leqslant k-1, \\
(k, k+1, k-1,2,3), & \alpha=k, \\
(n-\alpha+1, n-\alpha+1, \alpha-1, \alpha-k+1,2), & k+1 \leqslant \alpha \leqslant k+2, \\
(n-\alpha+1, n-\alpha+1, n-\alpha+3, \alpha-k+1, \alpha-k-1), & k+3 \leqslant \alpha \leqslant n-1, \\
(1,2,3, k+1, k-1), & \alpha=n,\end{cases} \\
& r\left(b_{\alpha} c_{\alpha} \mid W_{1}\right)=\left\{\begin{array}{l}
(1,1,3, k+1, k) \\
(\alpha, \alpha+1,4-\alpha, k-\alpha+2, k+1) \\
(\alpha, \alpha+1, \alpha-1, k-\alpha+2, k-\alpha+4) \\
(n-k+1, n-k+1, k, 1,3) \\
(n-k, n-k, k+1,3,2) \\
(n-k-1, n-k-1, n-k+1,4,1) \\
(n-\alpha+2, n-\alpha+2, n-\alpha+4, \alpha-k+1, \alpha-k-1)
\end{array}\right. \\
& \left(\begin{array}{ll}
(1,2,3, k+1, k), & \alpha=1, \\
(2,3,1, k, k+1), & \alpha=2,
\end{array}\right. \\
& r\left(b_{\alpha} c_{\alpha+1} \mid W_{1}\right)= \begin{cases}(\alpha, \alpha+1, \alpha-1, k-\alpha+2, k-\alpha+4), & 3 \leqslant \alpha \leqslant k-1, \\
(k, k+1, k-1,1,4), & \alpha=k, \\
(n-k+1, n-k+1, k, 2,3), & \alpha=k+1, \\
(n-k, n-k, k+1,3,1), & \alpha=k+2, \\
(n-\alpha+2, n-\alpha+2, n-\alpha+4, \alpha-k+1, \alpha-k-1), & k+3 \leqslant \alpha \leqslant n-1, \\
(2,1,4, k+1, k-1), & \alpha=n,\end{cases} \\
& r\left(c_{\alpha} d_{\alpha} \mid W_{1}\right)= \begin{cases}(2,0,4, k+2, k), & \alpha=1, \\
(2,3,3, k+1, k+1), & \alpha=2, \\
(3,4,0, k, k+2), & \alpha=3, \\
(\alpha, \alpha+1, \alpha-1, k-\alpha+3, k-\alpha+5), & 4 \leqslant \alpha \leqslant k, \\
(k+1, k+2, k, 0,4), & \alpha=k+1, \\
(n-k+1, n-k+1, k+1,3,3), & \alpha=k+2, \\
(n-k, n-k, n-k+2,4,0), & \alpha=k+3, \\
(n-\alpha+3, n-\alpha+3, n-\alpha+5, \alpha-k+1, \alpha-k-1), & k+4 \leqslant \alpha \leqslant n .\end{cases}
\end{aligned}
$$

From above representation we see that $r\left(b_{\alpha} c_{\alpha} \mid W_{1}\right)=r\left(b_{\alpha} c_{\alpha+1} \mid W_{1}\right)$ when $1 \leqslant \alpha \leqslant n$ and $\alpha \neq 1,2,3, k, k+$ $1, k+2, k+3, n$ and no other edges have same representation. If we take odd $\alpha$, where $1 \leqslant \alpha \leqslant n$ and $\alpha \neq 1,3, k+1, n-1$ such that $W_{E}=W_{1} \cup\left\{d_{\alpha}\right\}$ then $r\left(b_{\alpha} c_{\alpha} \mid W_{E}\right) \neq r\left(b_{\alpha} c_{\alpha+1} \mid W_{E}\right)$ which implies that $\operatorname{edim}\left(D_{\mathfrak{n}}^{t}\right) \leqslant\left\lceil\frac{\mathfrak{n}}{2}\right\rceil+1$. So from Lemma $4.3 W_{E}$ is an edge metric basis for $D_{\mathfrak{n}}^{t}$ and edim $\left(D_{\mathfrak{n}}^{t}\right)=\left\lceil\frac{\mathfrak{n}}{2}\right\rceil+1$.

Case (II) When $n \equiv 2(\bmod 4)$. We can write $n=2 k, k \geqslant 3$, and $k$ is odd. Let $W_{1}=\left\{a_{1}, d_{1}, d_{3}, d_{k+2}\right\}$. Next, we give representation of edges of $D_{n}^{t}$ with respect to $W_{1}$.

$$
\begin{aligned}
r\left(a_{\alpha} a_{\alpha+1} \mid W_{1}\right) & = \begin{cases}(\alpha-1, \alpha+2,3, k-\alpha+3), & 1 \leqslant \alpha \leqslant 2, \\
(\alpha-1, \alpha+2, \alpha, k-\alpha+3), & 3 \leqslant \alpha \leqslant k, \\
(k-1, k+1, k+1,3), & \alpha=k+1, \\
(n-\alpha, n-\alpha+2, n-\alpha+4, \alpha-k+1), & k+2 \leqslant \alpha \leqslant n-1, \\
(0,3,4, k+1), & \alpha=n,\end{cases} \\
r\left(a_{\alpha} b_{\alpha} \mid W_{1}\right) & = \begin{cases}(\alpha-1, \alpha+1,4-\alpha, k+1), & 1 \leqslant \alpha \leqslant 2, \\
(\alpha-1, \alpha+1, \alpha-1, k-\alpha+3), & 3 \leqslant \alpha \leqslant k, \\
(n-\alpha+1, n-\alpha+2, \alpha-1,2), & k+1 \leqslant \alpha \leqslant k+2, \\
(n-\alpha+1, n-\alpha+2, n-\alpha+4, \alpha-k), & k+3 \leqslant \alpha \leqslant n,\end{cases}
\end{aligned}
$$




$$
\begin{aligned}
& r\left(b_{\alpha} b_{\alpha+1} \mid W_{1}\right)= \begin{cases}(\alpha, \alpha+1,2, k-\alpha+2), & 1 \leqslant \alpha \leqslant 2, \\
(\alpha, \alpha+1, \alpha-1, k-\alpha+2), & 3 \leqslant \alpha \leqslant k, \\
(k, k, k, 2), & \alpha=k+1, \\
(n-\alpha+1, n-\alpha+1, n-\alpha+3, \alpha-k), & k+2 \leqslant \alpha \leqslant n-1, \\
(1,2,3, k), & \alpha=n,\end{cases} \\
& r\left(b_{\alpha} c_{\alpha} \mid W_{1}\right)= \begin{cases}(1,1,3, k+1), & \alpha=1, \\
(\alpha, \alpha+1,4-\alpha, k-\alpha+3), & 2 \leqslant \alpha \leqslant 3, \\
(\alpha, \alpha+1, \alpha-1, k-\alpha+3), & 4 \leqslant \alpha \leqslant k, \\
(n-\alpha+2, n-\alpha+2, \alpha-1, k-\alpha+3), & k+1 \leqslant \alpha \leqslant k+2, \\
(n-\alpha+2, n-\alpha+2, n-\alpha+4, \alpha-k), & k+3 \leqslant \alpha \leqslant n,\end{cases} \\
& r\left(b_{\alpha} c_{\alpha+1} \mid W_{1}\right)= \begin{cases}(1,2,3, k+1), & \alpha=1, \\
(\alpha, \alpha+1, \alpha-1, k-\alpha+3), & 2 \leqslant \alpha \leqslant k, \\
(n-\alpha+2, n-\alpha+2, \alpha-1, \alpha-k), & k+1 \leqslant \alpha \leqslant k+2, \\
(n-\alpha+2, n-\alpha+2, n-\alpha+4, \alpha-k), & k+3 \leqslant \alpha \leqslant n-1, \\
(2,1,4, k), & \alpha=n,\end{cases} \\
& r\left(c_{\alpha} d_{\alpha} \mid W_{1}\right)= \begin{cases}(2,0,4, k+1), & \alpha=1, \\
(2,3,3, k+2), & \alpha=2, \\
(3,4,0, k+1), & \alpha=3, \\
(\alpha, \alpha+1, \alpha-1, k-\alpha+4), & 4 \leqslant \alpha \leqslant k+1, \\
(n-k+1, n-k+1, k+1,0), & \alpha=k+2, \\
(n-\alpha+3, n-\alpha+3, n-\alpha+5, \alpha-k), & k+3 \leqslant \alpha \leqslant n .\end{cases}
\end{aligned}
$$

From above representation we see that $r\left(b_{\alpha} c_{\alpha} \mid W_{1}\right)=r\left(b_{\alpha} c_{\alpha+1} \mid W_{1}\right)$ when $1 \leqslant \alpha \leqslant n$ and $\alpha \neq 1,2,3, k+$ $1, k+2, n$ and no other edges have same representation. If we take odd $\alpha$, where $1 \leqslant \alpha \leqslant n$ and $\alpha \neq$ $1,3, k+2$ such that $W_{E}=W_{1} \cup\left\{d_{\alpha}\right\}$ then $r\left(b_{\alpha} c_{\alpha} \mid W_{E}\right) \neq r\left(b_{\alpha} c_{\alpha+1} \mid W_{E}\right)$ which implies that edim $\left(D_{n}^{t}\right) \leqslant$ $\left\lceil\frac{\mathfrak{n}}{2}\right\rceil+1$. So from Lemma $4.3 W_{E}$ is an edge metric basis for $D_{n}^{t}$ and $\operatorname{edim}\left(D_{n}^{t}\right)=\left\lceil\frac{n}{2}\right\rceil+1$.

Let $W_{E}=\left\{a_{1}, d_{1}, d_{3}, d_{5}, \ldots, d_{n-2}, d_{n}\right\}$. We will show that $W_{E}$ is an edge metric basis of $D_{n}^{t}$ in Cases (III) and (IV), respectively.

Case (III) When $n \equiv 1(\bmod 4)$. We can write $n=2 k+1, k \geqslant 2$, and $k$ is even. Let $W_{1}=\left\{a_{1}, d_{1}, d_{3}, d_{k+3}\right\}$. Next, we give representation of edges of $D_{n}^{t}$ with respect to $W_{1}$.

$$
\begin{aligned}
& r\left(a_{\alpha} a_{\alpha+1} \mid W_{1}\right)= \begin{cases}(\alpha-1, \alpha+2,3, k+2), & 1 \leqslant \alpha \leqslant 2, \\
(\alpha-1, \alpha+2, \alpha, k-\alpha+4), & 3 \leqslant \alpha \leqslant k, \\
(n-\alpha, n-\alpha+2, \alpha, 3), & k+1 \leqslant \alpha \leqslant k+2, \\
(n-\alpha, n-\alpha+2, n-\alpha+4, \alpha-k), & k+3 \leqslant \alpha \leqslant n-1, \\
(0,3,4, k+1), & \alpha=n,\end{cases} \\
& r\left(a_{\alpha} b_{\alpha} \mid W_{1}\right)= \begin{cases}(\alpha-1, \alpha+1,4-\alpha, k+\alpha), & 1 \leqslant \alpha \leqslant 2, \\
(\alpha-1, \alpha+1, \alpha-1, k-\alpha+4), & 3 \leqslant \alpha \leqslant k+1, \\
(k, k+1, k+1,2), & \alpha=k+2, \\
(n-\alpha+1, n-\alpha+2, n-\alpha+4, \alpha-k-1), & k+3 \leqslant \alpha \leqslant n,\end{cases} \\
& r\left(b_{\alpha} b_{\alpha+1} \mid W_{1}\right)= \begin{cases}(\alpha, \alpha+1,2, k+1), & 1 \leqslant \alpha \leqslant 2, \\
(\alpha, \alpha+1, \alpha-1, k-\alpha+3), & 3 \leqslant \alpha \leqslant k, \\
(n-\alpha+1, n-\alpha+1, \alpha-1,2), & k+1 \leqslant \alpha \leqslant k+2, \\
(n-\alpha+1, n-\alpha+1, n-\alpha+3, \alpha-k-1), & k+3 \leqslant \alpha \leqslant n-1, \\
(1,2,3, k), & \alpha=n,\end{cases}
\end{aligned}
$$




$$
\begin{aligned}
& r\left(b_{\alpha} c_{\alpha} \mid W_{1}\right)= \begin{cases}(1,1,3, k+1), & \alpha=1, \\
(\alpha, \alpha+1,4-\alpha, k-\alpha+4), & 2 \leqslant \alpha \leqslant 3, \\
(\alpha, \alpha+1, \alpha-1, k-\alpha+4), & 4 \leqslant \alpha \leqslant k+1, \\
(n-\alpha+2, n-\alpha+2, \alpha-1, k-\alpha+4), & k+2 \leqslant \alpha \leqslant k+3, \\
(n-\alpha+2, n-\alpha+2, n-\alpha+4, \alpha-k-1), & k+4 \leqslant \alpha \leqslant n,\end{cases} \\
& r\left(b_{\alpha} c_{\alpha+1} \mid W_{1}\right)= \begin{cases}(1,2,3, k+1), & \alpha=1, \\
(\alpha, \alpha+1, \alpha-1, k-\alpha+4), & 2 \leqslant \alpha \leqslant k+1, \\
(n-\alpha+2, n-\alpha+2, \alpha-1, \alpha-k-1), & k+2 \leqslant \alpha \leqslant k+3, \\
(n-\alpha+2, n-\alpha+2, n-\alpha+4, \alpha-k-1), & k+4 \leqslant \alpha \leqslant n-1, \\
(2,1,4, k), & \alpha=n,\end{cases} \\
& r\left(c_{\alpha} d_{\alpha} \mid W_{1}\right)= \begin{cases}(2,0,4, k+1), & \alpha=1, \\
(2,3,3, k+2), & \alpha=2, \\
(3,4,0, k+2), & \alpha=3, \\
(\alpha, \alpha+1, \alpha-1, k-\alpha+5), & 4 \leqslant \alpha \leqslant k+1, \\
(k+2, n-k+1, k+1,3), & \alpha=k+2, \\
(n-k, n-k, k+2,0), & \alpha=k+3, \\
(n-\alpha+3, n-\alpha+3, n-\alpha+5, \alpha-k-1), & k+4 \leqslant \alpha \leqslant n .\end{cases}
\end{aligned}
$$

From above representation we see that $r\left(b_{\alpha} c_{\alpha} \mid W_{1}\right)=r\left(b_{\alpha} c_{\alpha+1} \mid W_{1}\right)$ when $1 \leqslant \alpha \leqslant n$ and $\alpha \neq 1,2,3, k+$ $2, k+3, n$ and no other edges have same representation. If we take odd $\alpha$, where $1 \leqslant \alpha \leqslant n$ and $\alpha \neq$ $1,3, k+3$ such that $W_{E}=W_{1} \cup\left\{d_{\alpha}\right\}$ then $r\left(b_{\alpha} c_{\alpha} \mid W_{E}\right) \neq r\left(b_{\alpha} c_{\alpha+1} \mid W_{E}\right)$ which implies that edim $\left(D_{n}^{t}\right) \leqslant$ $\left\lceil\frac{\mathfrak{n}}{2}\right\rceil+1$. So from Lemma $4.3 W_{E}$ is an edge metric basis for $D_{\mathfrak{n}}^{t}$ and $\operatorname{edim}\left(D_{\mathfrak{n}}^{t}\right)=\left\lceil\frac{\mathfrak{n}}{2}\right\rceil+1$.

Case (IV) When $n \equiv 3(\bmod 4)$. We can write $n=2 k+1, k \geqslant 3$, and $k$ is odd. Let $W_{1}=\left\{a_{1}, d_{1}, d_{3}, d_{k+2}\right.$, $\left.d_{k+4}\right\}$. Next, we give representation of edges of $D_{n}^{t}$ with respect to $W_{1}$.

$$
\begin{gathered}
r\left(a_{\alpha} a_{\alpha+1} \mid W_{1}\right)= \begin{cases}(\alpha-1, \alpha+2,3, k-\alpha+3, \alpha+k), & 1 \leqslant \alpha \leqslant 2, \\
(\alpha-1, \alpha+2, \alpha, k-\alpha+3, k-\alpha+5), & 3 \leqslant \alpha \leqslant k, \\
(n-\alpha, n-\alpha+2, \alpha, 3, k-\alpha+5), & k+1 \leqslant \alpha \leqslant k+2, \\
(n-k-3, n-k-1, n-k+1,4,3), & \alpha=k+3, \\
(n-\alpha, n-\alpha+2, n-\alpha+4, \alpha-k+1, \alpha-k-1), & k+4 \leqslant \alpha \leqslant n-1, \\
(0,3,4, n-k+1, n-k-1), & \alpha=n,\end{cases} \\
r\left(a_{\alpha} b_{\alpha} \mid W_{1}\right)= \begin{cases}(\alpha-1, \alpha+1,4-\alpha, k-\alpha+3, k+\alpha-1), & 1 \leqslant \alpha \leqslant 2, \\
(\alpha-1, \alpha+1, \alpha-1, k-\alpha+3, k-\alpha+5), & 3 \leqslant \alpha \leqslant k+1, \\
(n-\alpha+1, n-\alpha+2, \alpha-1, \alpha-k, k-\alpha+5), & k+2 \leqslant \alpha \leqslant k+3, \\
(n-\alpha+1, n-\alpha+2, n-\alpha+4, \alpha-k, \alpha-k-2), & k+4 \leqslant \alpha \leqslant n, \\
r\left(b_{\alpha} b_{\alpha+1} \mid W_{1}\right)= \begin{cases}(\alpha, \alpha+1,2, k-\alpha+2, \alpha+k-1), & 1 \leqslant \alpha \leqslant 2, \\
(\alpha, \alpha+1, \alpha-1, k-\alpha+2, k-\alpha+4), & 3 \leqslant \alpha \leqslant k, \\
(k+1, n-k, k, 2,3), & \alpha=k+1, \\
(n-\alpha+1, n-\alpha+1, k+1, \alpha-k, 2), & k+2 \leqslant \alpha \leqslant k+3, \\
(1,2,3, n-k, n-k-2), & k+4 \leqslant \alpha \leqslant n-1,\end{cases} \\
(1,1,3, k+2, k), & \alpha=n, \\
(\alpha, \alpha+1,4-\alpha, k-\alpha+3, \alpha+k-1), & \alpha=1, \\
(\alpha, \alpha+1, \alpha-1, k-\alpha+3, k-\alpha+5), & 2 \leqslant \alpha \leqslant 3, \\
(n-k, n-k, k+1,1,3), & 4 \leqslant \alpha \leqslant k+1, \\
(n-\alpha+2, n-\alpha+2, n-\alpha+4, \alpha-k, k-\alpha+5), & k+3 \leqslant \alpha \leqslant k+4, \\
(n-\alpha+2, n-\alpha+2, n-\alpha+4, \alpha-k, \alpha-k-2), & k+5 \leqslant \alpha \leqslant n,\end{cases}
\end{gathered}
$$




$$
\begin{aligned}
& r\left(b_{\alpha} c_{\alpha+1} \mid W_{1}\right)= \begin{cases}(1,2,3, k+2, k), & \alpha=1, \\
(\alpha, \alpha+1, \alpha-1, k-\alpha+3, k+\alpha-1), & 2 \leqslant \alpha \leqslant 3, \\
(\alpha, \alpha+1, \alpha-1, k-\alpha+3, k-\alpha+5), & 4 \leqslant \alpha \leqslant k, \\
(k+1, n-\alpha+2, \alpha-1, \alpha-k, k-\alpha+5), & k+1 \leqslant \alpha \leqslant k+2, \\
(n-\alpha+2, n-\alpha+2, n-\alpha+4, \alpha-k, \alpha-k-2), & k+3 \leqslant \alpha \leqslant n-1, \\
(2,1,4, k+1, k-1), & \alpha=n,\end{cases} \\
& r\left(c_{\alpha} d_{\alpha} \mid W_{1}\right)= \begin{cases}(2,0,4, k+2, k), & \alpha=1, \\
(2,3,3, k+2, k+1), & \alpha=2, \\
(3,4,0, k+1, k+2), & \alpha=3, \\
(\alpha, \alpha+1, \alpha-1, k-\alpha+4, k-\alpha+6), & 4 \leqslant \alpha \leqslant k+1, \\
(n-k+1, n-k+1, k+1,0,4), & \alpha=k+2, \\
(n-k, n-k, k+2,3,3), & \alpha=k+3, \\
(n-k-1, n-k-1, n-k+1,4,0), & \alpha=k+4, \\
(n-\alpha+3, n-\alpha+3, n-\alpha+5, \alpha-k, \alpha-k-2), & k+5 \leqslant \alpha \leqslant n .\end{cases}
\end{aligned}
$$

From above representation we see that $r\left(b_{\alpha} c_{\alpha} \mid W_{1}\right)=r\left(b_{\alpha} c_{\alpha+1} \mid W_{1}\right)$ when $1 \leqslant \alpha \leqslant n$ and $\alpha \neq 1,2,3, k+$ $1, k+2, k+3, k+4, n$ and no other edges have same representation. If we take odd $\alpha$, where $1 \leqslant \alpha \leqslant n$ and $\alpha \neq 1,3, k+2, k+4$ such that $W_{E}=W_{1} \cup\left\{d_{\alpha}\right\}$ then $r\left(b_{\alpha} c_{\alpha} \mid W_{E}\right) \neq r\left(b_{\alpha} c_{\alpha+1} \mid W_{E}\right)$ which implies that edim $\left(D_{n}^{t}\right) \leqslant\left\lceil\frac{n}{2}\right\rceil+1$. So from Lemma $4.3 W_{E}$ is an edge metric basis for $D_{n}^{t}$ and $\operatorname{edim}\left(D_{n}^{t}\right)=$ $\left\lceil\frac{\mathfrak{n}}{2}\right\rceil+1$.

\section{Conclusion}

In this paper, we have computed the exact value of the edge metric dimension of flower graph $f_{n \times 3}$, the prism related graphs $D_{n}^{\prime}$ and $D_{n}^{t}$. It has been observed that the edge metric dimension of these graphs is greater than the metric dimension and we concluded that the edge metric dimension of $D_{n}^{\prime}$ is constant while $f_{n \times 3}$ and $D_{n}^{t}$ have unbounded edge metric dimensions.

\section{References}

[1] M. Ahsan, Z. Zahid, S. Zafar, Edge metric dimension of some classes of circulant graphs, Analele Stiintifice ale Universitatii Ovidius Constanta (in press). 1

[2] M. Ali, G. Ali, U. Ali, M. T. Rahim, On cycle related graphs with constant metric dimension, Open J. Discrete Math., 2 (2012), 21-23.

[3] J. Cáceres, C. Hernando, M. Mora, I. M. Pelayo, M. L. Puertas, C. Seara, D. R. Wood, On the metric dimension of cartesian products of graphs, SIAM J. Discrete Math., 21 (2007), 423-441. 1

[4] G. Chartrand, L. Eroh, M. A. Johnson, O. R. Oellermann, Resolvability in graphs and the metric dimension of a graph, Discrete Appl. Math., 105 (2000), 99-113. 1

[5] G. Chartrand, C. Poisson, P. Zhang, Resolvability and the upper dimension of graphs, Comput. Math. Appl., 39 (2000), 19-28. 1

[6] G. Chartrand, P. Zhang, The theory and applications of resolvability in graphs: a survey, Congr. Numer., 160 (2003), 47-68. 1

[7] V. Filipović, A. Kartelj, J. Kratica, Edge Metric Dimension of Some Generalized Petersen Graphs, Results Math., 74 (2019), 15 pages. 1

[8] I. Gutman, L. Pavlović, More on distance of line graphs, Graph Theory Notes New York, 33 (1997), 14-18.

[9] M. Hallaway, C. X. Kang, E. Yi, On metric dimension of permutation graphs, J. Comb. Optim., 28 (2014), 814-826. 1

[10] F. Harary, R. A. Melter, On the metric dimension of a graph, Ars Combin., 2 (1976), 191-195. 1

[11] M. Imran, A. Q. Baig, A. Ahmad, Families of plane graphs with constant metric dimension, Util. Math., 88 (2012), 43-57. 1, 1.3

[12] M. Johnson, Structure-activity maps for visualizing the graph variables arising in drug design, J. Biopharm. Stat., 3 (1993), 203-236. 1

[13] A. Kelenc, N. Tratnik, I. G. Yero, Uniquely identifying the edges of a graph: the edge metric dimension, Discrete Appl. Math., 251 (2018), 204-220. 1, 1.1, 1.2

[14] S. Khuller, B. Raghavachari, A. Rosenfeld, Landmarks in graphs, Discrete Appl. Math., 70 (1996), 217-229. 1 
[15] R. A. Melter, I. Tomescu, Metric bases in digital geometry, Comput. Vision Graphics Image Process., 25 (1984), 113-121. 1

[16] I. Peterin, I. G. Yero, Edge metric dimension of some graph operations, Bull. Malays. Math. Sci. Soc., 43 (2020), 24652477. 1

[17] A. Sebö, E. Tannier, On metric generators of graphs, Math. Oper. Res., 29 (2004), 383-393.

[18] P. J. Slater, Leaves of trees, Proceedings of the Sixth Southeastern Conference on Combinatorics, Graph Theory, and Computing (Florida Atlantic Univ., Boca Raton), 1975 (1975), 549-559. 1

[19] P. J. Slater, Dominating and reference sets in graphs, J. Math. Phys. Sci., 22 (1988), 445-455.

[20] S. Sultan, On the Metric Dimension and Minimal Doubly Resolving Sets of Families of Graphs, Ph.D. Thesis (G. C. University), Lahore, (2018).

[21] I. Tomescu, M. Imran, R-sets and metric dimension of necklace graphs, Appl. Math. Inf. Sci., 9 (2015), 63-67.

[22] Y. Z. Zhang, S. G. Gao, On the edge metric dimension of convex polytopes and its related graphs, J. Comb. Optim., 39 (2020), 334-350. 1

[23] N. Zubrilina, On the edge dimension of a graph, Discrete Math., 341 (2018), 2083-2088. 1 